\title{
Patent highlights
}

A snapshot of noteworthy recent developments in the patent literature of relevance to pharmaceutical and medical research and development.

WO/2014/101718

Methods and compositions for treating and/or preventing a disease or disorder associated with abnormally high level of mitochondria factor 4

Quin Y, Yang F, Hao J \& Zhu P Institute of Biophysics, Chinese Academy of Sciences (Beijing, China)

- cancer $\cdot$ mitochondria $\cdot$ transcriptional elongation factors

Elongation factor 4 (EF4, originally known as LepA) is one of the most conserved proteins across all known genomes. It backtranslocates the elongating ribosome, causing the translation machinery to move one codon backward along the nascent mRNA. Its biological significance in mammals has not been fully elucidated, although it is expected that EF4 helps maintaining translation fidelity under stress conditions like in bacteria and yeast [1,2]. Eukaryotic homologs have a potential mitochondrial targeting signal in their $\mathrm{N}$-termini, but it used to be unclear whether ET4 is needed there, and for what purpose. The answer seems to be respiratory function and cellular energy management. The inventors show that mtEF4 co-localizes with the mitochondrial respiratory chain complex IV subunit and while it maintains mitochondrial translation at catalytic concentrations, it is inhibitory at the stoichiometric level. They also show that mtEF4 is highly overexpressed in cancer cells. The anticancer agents of the invention can be antibodies, binding proteins or siRNAs that modify a nuclear factor that controls mtEF4 transcription or bind to its mitochondrial target sequence. For a closely related peer review paper from this team, see [3].

Published July 3, 2014

\section{WO/2014/104224}

PGC-1 $\beta$ protein function regulator, mitochondria function regulator, antiobesity agent, and screening method therefor

Nakajima T, Fujita H, Aratini S

\& Yagishita $\mathrm{N}$

Nakajima T (Japan)

\section{- mitochondria • PPARGC1 • SYVN1 protein, hu- man $\cdot$ transcription factors $\cdot$ ubiquitin-protein ligases}

This patent application connects two factors that previously were not thought to be related - the peroxisome proliferator-activated receptor- $\gamma$ coactivator $1 \beta$ (PGC- $1 \beta$; a transcriptional regulator of cellular energy metabolism [4]) and the ubiquitin ligase synoviolin, which is highly expressed in rheumatoid synovial cells and is implicated in the endoplasmic-reticulum-associated degradation pathway [5]. The inventors have found that synoviolin negatively regulates PGC-1 $\beta$, and that blocking the interaction of these proteins (with small molecules) or reducing the expression of synoviolin (with siRNAs) promotes fatty acid $\beta$-oxidation in fat tissue mitochondria. One compound that is reported to be effective is LS-102 (Pharmacopeia, Inc.; a selective inhibitor for E3 ubiquitin ligase activity of synoviolin), which doubled PGC-1 $\beta$ protein levels in the 3T3-L1 murine fat precursor cell line, although it did not alter its mRNA synthesis. LS-102 treatment $(50 \mathrm{mg} / \mathrm{kg})$ increased the
Hermann AM Mucke

H.M. Pharma Consultancy h.mucke@hmpharmacon.com

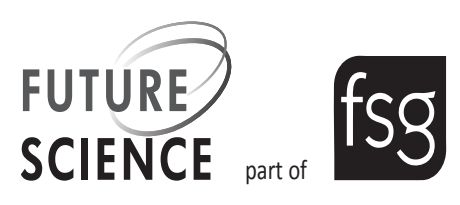


volume, density and number of mitochondria in adipose tissue of $\mathrm{C} 57 \mathrm{BL} / 6 \mathrm{~J}$ mice as well as $\beta$-oxidation of fatty acids. Other inhibitors are naphtalene derivatives, but quercetin is also effective. Note that inhibitors of small ubiquitin-like modifier E3 ligase activity suppress liver X receptor ligand-dependent transcriptional activation of the lipogenic program in hepatocytes [6], which points into the same direction.

Published July 3, 2014

\section{WO/2014/107745}

Metal-sensitive mutants of matrix metalloproteinases and uses thereof Paladini RD, Wei G \& Shepard HM Halozyme, Inc. (CA, USA)

- calcium • fibrosis $\cdot$ matrix metalloproteinases

The extracellular matrix (ECM) provides structural support for cells and tissues; excessive deposition of ECM components, such as collagen, can lead to fibrotic disease. The inventors have modified ECMdegrading matrix metalloproteinases (MMPs) and hyaluronidases in such a way that they become fully active only at extracellular calcium concentrations much higher than the physiological levels of 1.0-1.3 mM. Thus, unlike wild-type MMP polypeptides, the activity of csMMP polypeptides can be regulated by controlling local concentrations of calcium so that uncontrolled or prolonged degradation of collagen and other ECM components - a side effect of treatment with bacterial collagenases - is avoided. The lyophilized mutant MMPs (MMP-1, which cleaves the most abundant skin proteins collagen type I and III, is preferred) can be reconstituted in a buffer containing $10 \mathrm{mM}$ calcium. Following injection into the fibrotic tissue, the calcium steadily diffuses and the modified MMP becomes almost inactive. The MMPs of the invention typically contain one or more amino acid replacement at positions at or near the residues directly involved with zinc or calcium coordination. Efficacy was demonstrated with in vivo rat skin perfusion followed by western blotting, and by in vitro digestion of human keloidal collagen. The inventors claim utility for localized fibrotic conditions such as scars, Dupuytren's syndrome, Peyronie's disease, Ledderhose fibrosis or adhesive capsulitis ('frozen shoulder'). Note, however, that inhibition of MMP activity may be desired in preventing recurrent Dupuytren's contracture [7]. For information on metal-binding and catalytic sites of MMPs, see [8]. For the peer review companion paper, see [9].

Published July 10, 2014

\section{WO/2014/108520}

Benzylguanidine derivatives and therapeutic use for the treatment of protein misfolding diseases

Guedat P \& Bortolotti A

Medical Research Council (Swindon, UK)

- endoplasmic reticulum stress $\cdot$ eukaryotic initiation

factor- $2 \cdot$ guanabenz $\cdot$ proteostasis deficiencies

Guanabenz, an $\alpha 2$ receptor agonist, is an old antihypertensive drug that nowadays finds only very limited use in its original indication but has some very interesting alternative potential applications, including activity against prions [10] and protection of cells against stress resulting from protein misfolding [11]. The inventors have designed guanabenz derivatives that lack the undesireable $\alpha 2$ receptor agonism but retain the capability to block stress-induced dephosphorylation of the $\alpha$ subunit of eukaryotic translation initiation factor 2 (eIF $2 \alpha$ ). Earlier reports had indicated that the aryl group must be at least disubstituted in order for the compounds to exhibit useful pharmacological activity, but it is now evident that monosubstituted aryl derivatives are also active. Actually, 1-[(E)-[(2-chlorophenyl) methylidene ]amino]-guanidine, which is guanabenz with one of the two chlorine substituents removed, is the most preferred compound. The inventors claim to have tested for accumulation of mutant huntingtin amino-terminal fragment $(\mathrm{Htt} 48 \mathrm{Q})$ associated with Huntington's disease and SOD1 mutant $(\mathrm{A} 4 \mathrm{~V})$, associated with amyotrophic lateral sclerosis; of the P0S63del mutant causing Charcot-Marie-Tooth 1 $\mathrm{B}$ neuropathy; and for aggregation of rhodopsin $\mathrm{P} 23 \mathrm{H}$ which is associated with retinitis pigmentosa. However, no data are disclosed. For other guanabenz derivatives modified for anti-prion activity, see [12].

Published July 17, 2014

\section{WO/2014/110036}

SHIP1 modulators and methods related thereto Mackenzie LF, MacRury TB, Harwig C et al.

Aquinox Pharmaceuticals Inc. (British Columbia, Canada)

- allergy and immunology $\cdot$ naphthalenes $\cdot$ phosphoric monoester hydrolases

SHIP1 (an endogenous inhibitor of the phosphoinositide-3-kinase pathway) differs from SHIP2 and the $3^{\prime}$ inositol phosphatase phosphate and tensin homolog in that its expression is essentially restricted to immune and hematopoietic cells. Diminished SHIP1 activity or expression has been observed in human inflammatory diseases and hematopoietic malignancies [13], but its involvement is broader, extending for example to bone metabolism [14]. This 
application, which claims decahydronaphthalene derivatives and does not appear to cover the company's clinical-stage oral SHIP1 activator AQX-1125 (in development for asthma; [15-17]), continues from WO/2011/069118 which discloses tetracyclic SHIP1 modulators. Several compounds activated human SHIP1 in vitro by more than $65 \%$, and also blocked Akt phosphorylation. Two of these - one of which is $\mathrm{N}-[(2 \mathrm{R}, 3 \mathrm{~S}, 4 \mathrm{~S}, 4 \mathrm{aS}, 8 \mathrm{aS})-4-[(3,5$-dihydroxyphenyl) carbonyl]-3,4a, 8, 8-tetramethyl-decahydronaphthalene-2-yl]acetamide - inhibited allergen-induced passive cutaneous anaphylaxis in mice by more than $50 \%$ at $20 \mathrm{mg} / \mathrm{kg}$. The therapeutic claim (No. 23) is for autoimmune diseases, inflammation and neoplasms.

Published July 17, 2014

\section{WO/2014/112145}

Therapeutic agent and therapeutic method relating to 1,25D3-MARRS for neurological disease such as Alzheimer's disease

Tohda C

Resilo Co. Ltd (Tokyo, Japan)

- Alzheimer disease • diosgenin • PDIA3 protein, human - protein disulfide-isomerases $•$ vitamin D

1,25D3-MARRS stands for ' 1,25 dihydroxyvitamin D3 membrane associated, rapid response steroid-binding' protein, an alternate receptor for vitamin $\mathrm{D}$ also referred to as ERp57, GRp58 and PDIA3 [18,19]. The inventors have shown that diosgenin, a plant-derived steroidal sapogenin - known to be neuroprotective in retroviral infections but originally presumed to act as an antioxidant [20] - directly activates 1,25D3-MARRS. The same is true for the related denosomin, which promotes axon regeneration in spinal cord injury models [21]. Data from novel object recognition tests in a transgenic mouse strain $(5 \mathrm{xFAD})$ harboring five familial Alzheimer-related mutations are presented. Although the mechanism of this alternate vitamin D pathway has not yet been fully elucidated - protein kinase $\mathrm{C}$ activation and nuclear translocation of the bound receptor protein are certainly involved - it could shed new light on how vitamin D and its analogs exert their effects against Alzheimer's disease, which might be protective rather than therapeutic because results from clinical trials had mostly negative results. The natural products diosgenin and denosomin could serve as lead structures to develop drugs that do not bind to the classical vitamin $\mathrm{D}$ receptor, and which have more immediate effects on cognition. For the companion peer review papers, see [22,23].

Published July 24, 2014

\section{WO/2014/113450}

Decreasing inflammatory response in a blood vessel

Downey TJ, Utecht RE \& Munger KA

Alumend LLC (TX, USA)

- angioplasty, balloon, laser-assisted $\cdot$ atherosclerosis

- radiation-sensitizing agents

Larger blood vessels can become acutely inflamed as a result of infection, or by damage to the endothelium (e.g., from balloon angioplasty). Chronic inflammation is prominently exemplified by atherosclerosis where the damage caused by the endothelial uptake of oxidized low-density lipoproteins triggers a cascade of immune responses, which eventually result in atheroma formation. The inventors have used 4-amino-1,8-naphthalimides (a group of compounds known to be chemically activated by radiation to form condensation products with proteins via their amino moiety) to treat such conditions. The core structure of these compounds is an inhibitor of poly(ADP-ribose) polymerase [24] and telomerase [25]. The delivery system contains fiber optics in its lumen to irradiate the target zone with blue light at low power while the vessel is perfused. With co-administration of the compounds and photoactivation in a balloon vessel injury model, rat femoral arteries developed little or no intimal thickening after 2 weeks. It is suggested that vascular compliance was also improved, but no data are presented in support. Some of the company's prior patents, which originated at PhotoBioMed Corp., claimed naphthalimide-labeled chitosan for photoangioplasty, tethering pharmaceutical agents to tissue surfaces to provide local drug delivery, and for chemically bonding skin care products (see, e.g., WO/2005/044309).

Published July 24, 2014

\section{WO/2014/114644}

Use of Tungsten $(\mathrm{VI})$ salts for the treatment of female infertility in non-diabetic mammals

Canal Amalzán I \& Arbat Bugié A

Oxolife S.L. (Sant Quirze Del Vallès, Spain)

- anovulation • infertility, female $\cdot$ sodium tungstate(VI)

There are very few medical applications for tungsten salts, but Spanish researchers seem to have taken particular interest recently, as evidenced by WO/2014/096498 claiming tungstate salts as anti-platelet agents (reviewed in Pharm Patent Analyst 3(5)); earlier examples include WO/2002/098435 for obesity and EP-755681 for diabetes. It has been reported that sodium tungstate can reverse the loss of fertility in the standard rat model of streptozotocin-induced diabetes in male [26] as well as in female [27] animals. According to the data presented 
here, the effect of tungsten on female fertility is independent of diabetes: in IRS2 knockout mice, whose females have reduced follicular development and only $9 \%$ of the wildtype's fertility but remain relatively euglycemic during youth, sodium tungstate $(2 \mathrm{mg} / \mathrm{ml}$ in drinking water; about about $180 \mathrm{mg} / \mathrm{kg} /$ day) restored the normal estrous cycle. The question is, at what price? The major site of tungsten deposition in mice after 16 weeks of uptake in drinking water is bone, where it has a clear effect on DNA damage and pre-leukemic B-cell formation in bone marrow [28]. In humans, elevated tungsten concentrations are strongly associated with the prevalence of stroke [29]. However, none of the beneficial effects of tungsten salts that has been reported has found a proper explanation to date, which makes such reports extremely interesting.

Published July 31, 2014

\section{WO/2014/114779}

Substituted phtalamic acids as sortilin inhibitors

Maltas PJ, Watson S, Langgard M \& David L

H. Lundbeck A/S (Valby, Denmark)

- adaptor proteins $\cdot$ nerve degeneration $\cdot$ nerve growth

factors $\cdot$ vesicular transport

Sortilin (NTR-3, GP95) is a widely expressed type I membrane receptor that was originally identified as a sorting receptor in Saccharomyces cerevisiae. It mediates pro-apoptotic effects of pro-neurotrophins, and is considered a key regulator of the balance between generally opposing effects of mature and pro-forms of neurotrophins [30]. Sortilin/P75-mediated apoptosis has been suggested as a neuroprotective target mechanism in neurodegenerative disorders. The compounds that are disclosed here are among the first small molecule inhibitors of sortilin interaction; they cover Lundbeck's current lead, AF38469 (N-(6-methyl-pyridin2-yl)-5-trifluoromethyl-phthalamic acid; Example 1 in the application) [31] but not AF40431 which the company had described earlier [32]. The presented data show results from affinity assays based on the displacement of $3 \mathrm{H}$-neurotensin from human sortilin and from homogeneous time-resolved fluorescence assays measuring inhibition of proNGF and progranulin binding to sortilin. The claims cover almost every known neurodegenerative disease; but how inhibition of neurotrophic factor binding to sortilin - which apparently is a node in several pathways - would affect essential mechanisms of neuronal maintenance and regeneration is not known yet.

Published July 31, 2014
WO/2014/120569

Methods and compositions for human

epididymis protein-4 (HE4)

Kalluri R \& Lebleu VS

Beth Israel Deaconess Medical Center, Inc. (MA, USA)

- fibrosis - glomerular filtration rate • kidney diseases

Most chronic renal damage, irrespective of etiology, leads to renal fibrosis, a self-perpetuating process of collagen deposition that is facilitated by the recruitment of activated fibroblasts and the progression of an inflammatory response. One of the most overexpressed proteins in fibrotic kidneys is the human epididymis protein-4 (HE4), a glycoprotein of unknown biological function that has recently been proven to be a reliable serum cancer biomarker especially for gynecological malignancies [33], and it probably is an anticancer agent [34]. The results presented here confirm that HE4 is a pan-serine protease inhibitor (as had been expected because of its four-disulfide WAP core domain repeats), specifically of matrix metalloproteinases 2 and 9, that prevents degradation of type I collagen. In fibrotic kidneys of aSMA-RFP transgenic mice subjected to unilateral ureteral obstruction, treatment with a neutralizing antibody to HE4 (described in U.S. Patent 7,846,692) improved not only renal histology but also functional parameters (blood urea nitrogen and urine albumin-tocreatinine ratio). Claims extend to non-antibody binding proteins, interfering RNA expression inhibitors, and other types of organ fibrosis. The invention also features HE4 and two specific serine proteases, Prss 23 and Prss35, as a diagnostic marker for organ fibrosis or a risk of developing organ fibrosis. The nature of HE4 as a broad-spectrum protease inhibitor had been described before the patent application's priority date [35]. For the peer review companion paper, see [36].

Published August 7, 2014

\section{WO/2014/120885}

Treatment of drug abuse by preventing GAPDH nitrosylation

Snyder SH, Sen N \& Xu R

The Johns Hopkins University (MD, USA)

- glyceraldehyde-3-phosphate dehydrogenase

(phosphorylating) • selegiline $\cdot$ ubiquitin-protein ligases

Glyceraldehyde-3-phosphate dehydrogenase (GAPDH), a key enzyme in glycolysis, can undergo S-nitrosylation, which facilitates its binding to the E3 ubiquitin ligase, Siah1. The GAPDH-Siah protein complex translocates to the nucleus and triggers apoptosis [37]. It was known that the neuroprotective effects of $\mathrm{R}(-)$ deprenyl, a monoamine oxidase B (MAO-B) inhibitor 
used in early-stage Parkinson's disease, was at least in part attributable to its ability to prevent $S$-nitrosylation of GAPDH [38]. The inventors report that this same signaling cascade mediates at least some of the transcriptional and behavioral actions of cocaine, R-(-)-deprenyl, but particularly that of the related drug CGP3466B which is devoid of MAO-B inhibition. It prevents the enhancement of the cAMP response element-binding protein signaling system caused by lower, behavioral-stimulant doses of cocaine, and also the stimulation of the $\mathrm{p} 53$ cytotoxic system by higher, neurotoxic doses. CGP3466B $(0.15$ $\mathrm{mg} / \mathrm{kg}$ i.p.) substantially reduced locomotor stimulation by cocaine in mice moving in an open field paradigm, without effect on basal locomotor activity. In contrast, the MAO-B inhibitors rasagiline, pargyline and lazabemide, when combined with cocaine, elicited greater locomotor stimulation than cocaine alone and did not prevent cocaine-induced neurotoxicity. Pretreatment with CGP3466B also reduced conditioned placed preference, a measure of cocaine seeking behavior. This could open new avenues in the treatment of stimulant dependence. For the peer review companion paper, see [39].

\section{Published August 7, 2014}

\section{WO/2014/123882}

\section{2,6,7 Substituted purines as HDM2 inhibitors Christopher MP, Fradera Llinas FX, Machacek M et al. \\ Merck Sharp \& Dohme Corp. (NJ, USA)}

- neoplasms • proto-oncogene proteins c-mdm2

Human double minute 2 (HDM2), an E3 ubiquitin-protein ligase, is a negative autoregulator of

\section{References}

1 Qin Y, Polacek N, Vesper O et al. The highly conserved LepA is a ribosomal elongation factor that back-translocates the ribosome. Cell 127(4), 721-733 (2006).

2 Zhang D, Qin Y. The paradox of elongation factor 4: highly conserved, yet of no physiological significance? Biochem. J. 452(2), 173-181 (2013).

3 Yang F, Gao Y, Li Z et al. Mitochondrial EF4 links respiratory dysfunction and cytoplasmic translation in Caenorhabditis elegans. Biochim. Biophys. Acta 15 (2014).

4 Lai L, Wang M, Martin OJ et al. A role for peroxisome proliferator-activated receptor $\gamma$ coactivator 1 (PGC-1) in the regulation of cardiac mitochondrial phospholipid biosynthesis. J. Biol. Chem. 289(4), 2250-2259 (2014).

5 Yagishita N, Aratani S, Leach C et al. RING-finger type E3 ubiquitin ligase inhibitors as novel candidates for the the classical tumor suppressor protein, $\mathrm{p} 53$. In the absence of oncogenic stress signals, it binds to p53 and blocks its ability to activate transcription, while in the presence of such signals HDM2 lets $\mathrm{p} 53$ activity rise to induce cell cycle arrest and apoptosis [40]. Restoring the function of $\mathrm{p} 53$ in tumor cells by antiHDM2 therapy should therefore result in slowing the tumor proliferation. Several classes of small-molecule inhibitors of HDM2-p53 interaction have been described, based on piperidines [41], spiro-oxindoles [42] and diphenylpyrroles [43]. Only two candidate drugs from Novartis, CGM097 and HDM201 have reached Phase I clinical trials. The present application introduces a new such class, 2,6,7 substituted purines. A large amount of synthesis and chemical characterization data is presented but not the slightest indication of biological data is shared, not even on a categorical scale. This underscores the competitiveness of this field. It is not to be expected that HDM2 inhibitors could become stand-alone oncology therapeutics; but at some point they might be useful to prevent cancer recurrence, or - in combination with cytotoxic drugs - slow metastasis.

\section{Published August 14, 2014}

\section{Financial \& competing interests disclosure}

The author has no relevant affiliations or financial involvement with any organization or entity with a financial interest in or financial conflict with the subject matter or materials discussed in the manuscript. This includes employment, consultancies, honoraria, stock ownership or options, expert testimony, grants or patents received or pending, or royalties.

No writing assistance was utilized in the production of this manuscript.

treatment of rheumatoid arthritis. Int. J. Mol. Med. 30(6), 1281-1286 (2012).

6 Zhang Y, Gan Z, Huang P et al. A role for protein inhibitor of activated STAT1 (PIAS1) in lipogenic regulation through SUMOylation-independent suppression of liver X receptors. J. Biol. Chem. 287(45), 37973-37985 (2012).

7 Townley WA, Cambrey AD, Khaw PT et al. Matrix metalloproteinase inhibition reduces contraction by dupuytren fibroblasts. J. Hand Surg. Am. 33(9), 1608-1616 (2008).

8 Maskos K. Crystal structures of MMPs in complex with physiological and pharmacological inhibitors. Biochimie 87(3-4), 249-263 (2005).

9 Paladini RD, Wei G, Kundu A et al. Mutations in the catalytic domain of human matrix metalloproteinase-1 (MMP-1) that allow for regulated activity through the use of Ca2+. J. Biol. Chem. 288(9), 6629-6639 (2013). 
10 Tribouillard-Tanvier D, Béringue V, Desban N et al. Antihypertensive drug guanabenz is active in vivo against both yeast and mammalian prions. PLoS ONE 3(4), e1981 (2008).

11 Tsaytler P, Harding HP, Ron D, Bertolotti A. Selective inhibition of a regulatory subunit of protein phosphatase 1 restores proteostasis. Science 332 (6025), 91-94 (2011).

12 Gug F, Oumata N, Tribouillard-Tanvier D et al. Synthesis of conjugates of 6-aminophenanthridine and guanabenz, two structurally unrelated prion inhibitors, for the determination of their cellular targets by affinity chromatography. Bioconjug. Chem. 21(2), 279-288 (2010).

13 Fernandes S, Iyer S, Kerr WG. Role of SHIP1 in cancer and mucosal inflammation. Ann. N. Y. Acad. Sci. 1280, 6-10 (2013).

14 Iyer S, Margulies BS, Kerr WG. Role of SHIP1 in bone biology. Ann. N. Y. Acad. Sci. 1280, 11-14 (2013).

15 Stenton GR, Mackenzie LF, Tam P et al. Characterization of AQX-1125, a small-molecule SHIP1 activator: Part 1. Effects on inflammatory cell activation and chemotaxis in vitro and pharmacokinetic characterization in vivo. Br. J. Pharmacol. 168(6), 1506-1518 (2013).

16 Stenton GR, Mackenzie LF, Tam P et al. Characterization of AQX-1125, a small-molecule SHIP1 activator: Part 2. Efficacy studies in allergic and pulmonary inflammation models in vivo. Br. J. Pharmacol. 168(6), 1519-1529 (2013).

17 Leaker BR, Barnes PJ, O'Connor BJ et al. The effects of the novel SHIP1 activator AQX-1125 on allergen-induced responses in mild to moderate asthma. Clin. Exp. Allergy 15, doi:10.1111/cea.12370 (2014) (Epub ahead of print).

18 Nemere I, Safford SE, Rohe B et al. Identification and characterization of 1,25D3-membrane-associated rapid response, steroid (1,25D3-MARRS) binding protein. J. Steroid Biochem. Mol. Biol. 89-90(1-5), 281-285 (2004).

19 Nemere I, Farach-Carson MC, Rohe B et al. Ribozyme knockdown functionally links a 1,25( $\mathrm{OH}) 2 \mathrm{D} 3$ membrane binding protein (1,25D3-MARRS) and phosphate uptake in intestinal cells. Proc. Natl Acad. Sci. USA 101(19), 7392-7397 (2004).

20 Turchan-Cholewo J, Liu Y, Gartner S et al. Increased vulnerability of ApoE4 neurons to HIV proteins and opiates: protection by diosgenin and L-deprenyl. Neurobiol. Dis. 23(1), 109-119 (2006).

21 Teshigawara K, Kuboyama T, Shigyo M et al. A novel compound, denosomin, ameliorates spinal cord injury via axonal growth associated with astrocyte-secreted vimentin. $\mathrm{Br}$. J. Pharmacol. 168(4), 903-919 (2013).

22 Tohda C, Urano T, Umezaki M et al. Diosgenin is an exogenous activator of $1,25 \mathrm{D}_{3}$-MARRS/Pdia3/ERp57 and improves Alzheimer's disease pathologies in 5XFAD mice. Sci. Rep. 2, 535 (2012).

23 Tohda C, Lee YA, Goto Y, Nemere I. Diosgenin-induced cognitive enhancement in normal mice is mediated by $1,25 \mathrm{D}_{3}$ MARRS. Sci. Rep. 3, 3395 (2013).

24 Schlicker A, Peschke P, Bürkle A et al. 4-Amino-1,8naphthalimide: a novel inhibitor of poly(ADP-ribose) polymerase and radiation sensitizer. Int. J. Radiat. Biol. 75(1), 91-100 (1999).
25 Ghosh U, Bhattacharyya NP. Benzamide and 4-amino 1,8 naphthalimide treatment inhibit telomerase activity by down-regulating the expression of telomerase associated protein and inhibiting the poly(ADP-ribosyl)ation of telomerase reverse transcriptase in cultured cells. FEBS J. 272(16), 4237-4248 (2005).

26 Ballester J, Domínguez J, Muñoz MC et al. Tungstate treatment improves Leydig cell function in streptozotocindiabetic rats. J. Androl. 26(6), 706-715 (2005).

27 Ballester J, Muñoz MC, Domínguez J et al. Tungstate administration improves the sexual and reproductive function in female rats with streptozotocin-induced diabetes. Hum. Reprod. 22(8), 2128-2135 (2007).

28 Kelly AD, Lemaire M, Young YK et al. In vivo tungsten exposure alters B-cell development and increases DNA damage in murine bone marrow. Toxicol. Sci. 131(2), 434-446 (2013).

29 Tyrrell J, Galloway TS, Abo-Zaid G et al. High urinary tungsten concentration is associated with stroke in the National Health and Nutrition Examination Survey 1999-2010. PLoS ONE 8(11), e77546 (2013).

30 Nykjaer A, Willnow TE. Sortilin: a receptor to regulate neuronal viability and function. Trends Neurosci. 35(4), 261-270 (2012).

31 SchrØder TJ, Christensen S, Lindberg $S$ et al. The identification of AF38469: an orally bioavailable inhibitor of the VPS10P family sorting receptor Sortilin. Bioorg. Med. Chem. Lett. 24(1), 177-180 (2014).

32 Andersen JL, SchrØder TJ, Christensen S et al. Identification of the first small-molecule ligand of the neuronal receptor sortilin and structure determination of the receptor-ligand complex. Acta Crystallogr. D. Biol. Crystallogr. 70(Pt 2), 451-460 (2014).

33 Speeckaert MM, Speeckaert R, Delanghe JR. Human epididymis protein 4 in cancer diagnostics: a promising and reliable tumor marker. Adv. Clin. Chem. 59, 1-21 (2013).

34 Kong X, Chang X, Cheng H et al. Human epididymis protein 4 inhibits proliferation of human ovarian cancer cells via the mitogen-activated protein kinase and phosphoinositide 3-kinase/AKT pathways. Int. J. Gynecol. Cancer 24(3), 427-436 (2014).

35 Chhikara N, Saraswat M, Tomar AK et al. Human epididymis protein-4 (HE-4): a novel cross-class protease inhibitor. PLoS ONE 7(11), e 47672 (2012).

36 LeBleu VS, Teng Y, O'Connell JT et al. Identification of human epididymis protein- 4 as a fibroblast-derived mediator of fibrosis. Nat. Med. 19(2), 227-231 (2013).

37 Hara MR, Agrawal N, Kim SF et al. S-nitrosylated GAPDH initiates apoptotic cell death by nuclear translocation following Siah1 binding. Nat. Cell Biol. 7(7), 665-674 (2005).

38 Hara MR, Thomas B, Cascio MB et al. Neuroprotection by pharmacologic blockade of the GAPDH death cascade. Proc. Natl Acad. Sci. USA 103(10), 3887-3889 (2006).

$39 \mathrm{Xu} \mathrm{R}$, Serritella AV, Sen T et al. Behavioral effects of cocaine mediated by nitric oxide-GAPDH transcriptional signaling. Neuron 78(4), 623-630 (2013). 
40 Böttger A, Böttger V, Garcia-Echeverria C et al. Molecular characterization of the hdm2-p53 interaction. J. Mol. Biol. 269(5), 744-756 (1997).

41 Ma Y, Lahue BR, Shipps GW Jr et al. Substituted piperidines as HDM2 inhibitors. Bioorg. Med. Chem. Lett. 24(4), 1026-1030 (2014).
42 Zou P, Zheng N, Yu Y et al. Preclinical pharmacokinetics of MI-219, a novel human double minute 2 (HDM2) inhibitor and prediction of human pharmacokinetics. J. Pharm. Pharm. Sci. 15(2), 265-280 (2012).

43 Gomha SM, Eldebss TM, Abdulla MM et al. Diphenylpyrroles: Novel p53 activators. Eur. J. Med. Chem. 82, 472-479 (2014). 